\section{Newborn Tongue-tie and Breast-Feeding}

To the Editor: I read the article titled "Newborn Tonguetie: Prevalence and Effect on Breast-Feeding" that appeared the January-February 2005 issue. ${ }^{1}$ I am the author of the Assessment Tool for Lingual Frenulum Function (ATLFF) referred to in this article. There are several substantial factual errors in this article that require correction.

1. The authors state that "One of the three investigators examined the tongues of all babies thought by the nurses' initial screening to have the appearance of tongue-tie," and "Although the ATLFF is not designed to be used with normal infants, all the function items and some of the appearance items can be tested on normal infants."

The ATLFF is a screening tool. It was designed to be used on ALL infants under three months of age to identify those that are tongue-tied and those that are not.

2. I came to Regions Hospital to provide training; however, the hospital's resources were limited, and I spent less than 2 hours scoring only 2 babies as a demonstration to the researchers. None of the investigators in this study demonstrated proficiency in the use of this screening tool. This hardly constitutes a training standard on which to base an inter-rater reliability study.

3 . The scoring parameters are incorrectly stated in this article. A perfect score on the function items is 14, regardless of the appearance item score; an 11 on the function items is acceptable if the appearance item score is 10. A function item score of less than 11 means the infant's tongue function is impaired and the infant is tongue-tied. Treatment recommendations are: when the function item score is less than 11 and the appearance item score is between 8 and 10, frenotomy should be considered if management fails; frenotomy is necessary if the function item score is less than 11 and the appearance item score is less than 8 . The function items are considered more important as an indicator of the presence of tongue-tie in this scoring system. Appearance deficits alone are not an indicator of the presence of tongue-tie and therefore should not be used as a selection criterion for further screening or for treatment!

The authors state that "Twelve of the tongue-tied infants had ATLFF scores of perfect, none had scores of acceptable, and 6 had scores of Function impaired." By definition, no tongue-tied infant can earn a perfect score on this screening tool. If an infant has a perfect scores, he or she is not tongue-tied.

4. Because the ATLFF is a screening tool, it is insufficient to be used as a predictor of breast-feeding outcomes. I am not surprised that "[ $t$ ]he ATLFF was not a useful tool to identify which tongue-tied infants are at risk for breast-feeding problems." Breast-feeding is a complex set of behaviors involving 2 people. My tool identifies only the deficits of those babies who have difficulty with one aspect of the breast-feeding relationship: the function of the tongue as a result of tongue-tie.

These substantial factual errors compel one to question the integrity of this study's findings.

Alison K. Hazelbaker, MA, IBCLC

Private practice Columbus, Ohio

\section{References}

1. Ricke LA, Baker NJ, Madlon-Kay DJ, DeFor TA. Newborn tongue-tie: prevalence and effect on breast-feeding. J Am Board Fam Pract 2005;18:1-7.

Author's Reply

To the Editor: We appreciate the opportunity to reply to Alison Hazelbaker's letter regarding our article, "Newborn Tongue-tie: Prevalence and Effect on Breast-Feeding" in the January-February issue of JABFP.

1. Four of the 5 appearance items in the ATLFF involve scoring the lingual frenulum. Many newborns have no visible lingual frenulum. We concluded, therefore, apparently incorrectly, that the ATLFF was designed to be used only on infants with the appearance of tongue-tie. We apologize for our error.

2. Because we knew our training time with Ms. Hazelbaker was limited, we videotaped her examining 4 babies (not 2 as stated in her letter.) We reviewed the videotape on later occasions and also contacted Ms. Hazelbaker with scoring questions.

3. The scoring information included in our article is correct but incomplete. Because our study did not include treatment decisions (ie, frenotomy), we did not include the portions of the scoring system related to management in our article. In our study, infants were identified as tongue-tied based on appearance only, as stated under Methods.

4. Ms. Hazelbaker had a copy of our study protocol. One of our study goals clearly was to test the usefulness of the ATLFF in identifying which tongue-tied infants were at risk for breast-feeding problems. She indicated support of our study by spending a day at our hospital educating our nursery nurses, training us, and corresponding with us when we had questions about the ATLFF. We were unaware of any objections to the study goals or methods.

We were surprised and disappointed that the ATLFF did not turn out to be a useful tool. We greatly respect 